\title{
ON MULTIPLICITY OF SPECTRUM FOR ANDERSON TYPE OPERATORS WITH HIGHER RANK PERTURBATIONS
}

\author{
ANish MALLiCK AND P. A. NARAYANAN
}

\begin{abstract}
Here, we focus on Anderson type operators over infinite graphs where the randomness acts through higher rank perturbations. We show that for special family of graphs, the operator has non-trivial multiplicity for its pure point spectrum. We, also, show that for some family of graphs, any unitary which fixes the random operator, arising from an automorphism of the graph is identity; but that, for these graphs the spectrum of the random operator has non-trivial multiplicity.
\end{abstract}

Mathematics subject classification (2010): 47A10, 47B39, 81Q10.

Keywords and phrases: Anderson model, spectrum, difference operator.

\section{REFERENCES}

[1] Michael Aizenman And Simone Warzel, The canopy graph and level statistics for random operators on trees, Math. Phys. Anal. Geom., 9(4):291-333 (2007), 2006.

[2] Philip W Anderson, Absence of diffusion in certain random lattices, Physical review, 109(5):1492, 1958.

[3] M ANish AND Dhriti RANJAn Dolai, Multiplicity theorem of singular spectrum for general anderson type hamiltonian, arXiv:1709.01774, 2017.

[4] René Carmona And Jean Lacroix, Spectral theory of random Schrödinger operators, Springer Science \& Business Media, 2012.

[5] AdRian Dietlein AND AleXANDeR Elgart, Level spacing for continuum random schrödinger operators with applications, arXiv preprint arXiv:1712.03925, 2017.

[6] Peter D Hislop, Lectures on random Schrödinger operators, Fourth Summer School in Analysis and Mathematical Physics, 476:41-131, 2008.

[7] PETER D Hislop AND M KRISHNA, Eigenvalue statistics for random schrödinger operators with non rank one perturbations, Communications in Mathematical Physics, 340(1):125-143, 2015.

[8] VoJKAn JAKŠIĆ AND Yoram LAST, Spectral structure of anderson type hamiltonians, Inventiones mathematicae, 141(3):561-577, 2000.

[9] VOJKAN JAKŠIĆ AND YORAM LAST, Simplicity of singular spectrum in anderson-type hamiltonians, Duke Mathematical Journal, 133(1):185-204, 052006.

[10] Werner KIRSCH, An invitation to random Schrödinger operators. with an appendix by frédéric klopp, Panor. Synthéses. Random Schrödinger operators. Soc. Math. France, Paris, pages 1-119, 2008.

[11] Abel Klein And Stanislav Molchanov, Simplicity of eigenvalues in the anderson model, Journal of statistical physics, 122(1):95-99, 2006.

[12] ANISH MALlicK, Jakšić-last theorem for higher rank perturbations, Mathematische Nachrichten, 289(11-12):1548-1559, 2016.

[13] ANISH MALLICK, Multiplicity bound of singular spectrum for higher rank anderson models, Journal of Functional Analysis, 272(12):5162 - 5190, 2017.

[14] Sergey Naboko, Roger Nichols, and GÜnter Stolz, Simplicity of eigenvalues in andersontype models, Arkiv för Matematik, 51(1):157-183, 2013.

[15] P A NARAYANAN, Eigenvalue statistics for higher rank anderson model over canopy tree, arXiv preprint arXiv:1706.02488, 2017. 
[16] Christian Sadel and Hermann Schulz-Baldes, Random dirac operators with time reversal symmetry, Communications in Mathematical Physics, 295(1):209-242, 2010.

[17] BARRY Simon, Cyclic vectors in the anderson model, Reviews in Mathematical Physics, 6(05a):1183-1185, 1994. 